Natural Hazards and Earth System Sciences (2001) 1: 99-104

(C) European Geophysical Society 2001

\title{
Possible earthquake precursors revealed by LF radio signals
}

\author{
P. F. Biagi ${ }^{1}$, R. Piccolo ${ }^{1}$, A. Ermini ${ }^{2}$, S. Martellucci ${ }^{3}$, C. Bellecci ${ }^{3}$, M. Hayakawa ${ }^{4}$, V. Capozzi ${ }^{5}$, and S. P. Kingsley \\ ${ }^{1}$ Dept. of Physics, University of Bari, 173-70126 Bari, Italy \\ ${ }^{2}$ Dept. of Physics and Energy Science and Technology, University of Roma, 00133 Rome, Italy \\ ${ }^{3}$ INFM-Dept. of Physics and Energy Science and Technology, University of Roma, 00133 Rome, Italy \\ ${ }^{4}$ Dept. of Electronic Engineering, University of Electro-Communications, Chofu City, Tokyo 182-8585, Japan \\ ${ }^{5}$ Medicin Faculty and National Institute of Condensed Matter, University of Foggia, 71100 Foggia, Italy \\ ${ }^{6}$ Sheffield Centre for Earth Observation Science, University of Sheffield, Hicks Building, Sheffield S3 7RH, UK
}

Received: 27 April 2001 - Accepted: 23 August 2001

\begin{abstract}
Among radio signals, low frequency (LF) radio signals lie in the band between $30-300 \mathrm{kHz}$. Monitoring equipment with the ability to measure the electric strength of such signals at field sites, were designed and assembled in Italy. From 1993 onwards, the electric field strength of the MCO $(216 \mathrm{kHz}$, France) broadcasting station has been collecting measurements at two sites in central Italy that were chosen according to very low noise levels. At the end of 1996, radio signals from the CLT $(189 \mathrm{kHz}$, Italy) and CZE $(270 \mathrm{kHz}$, Czech Republic) broadcasting stations were included in the measurements. Meteorological data from central Italy were also collected over the same time period in order to study the influence of weather conditions on the experimental measurements. During the monitoring period, we observed some evident attenuation of the electric field strength in some of the radio signals at some of the receivers. The duration of the attenuation observed was several days, so it could possibly be related to particular meteorological conditions. On the other hand, this phenomenon might represent precursors of moderate $(3.0 \leq \mathrm{M} \leq 3.5)$ earthquakes that occurred near the receivers (within $50 \mathrm{~km}$ ) along the transmitter-receiver path. In this case, it is possible that the pre-seismic processes could have produced irregularities in the troposphere, such as ducts, reflecting layers and scattering zones, so that some local troposphere defocusing of the radio signals might have occurred. These observations were related only to moderate earthquakes and in these cases, suitable meteorological conditions were probably needed to observe the effect. Between February - March 1998, we observed at one measuring site, a significant increase in the CZE electric field strength. Unfortunately, we could not use the data of the other receiver in this case, due to frequent interruptions in the data set. The increase might have been a precursor of the strong seismic sequence $(M=5.0-6.0)$ that
\end{abstract}

Correspondence to: P. F. Biagi (biagi@ fisica.uniba.it) occurred during March - May 1998 in Slovenia at a location over $400 \mathrm{~km}$ from the receiver, but lying in the middle of the transmitter-receiver path. In this case, it is possible that an ionospheric disturbance, produced by the pre-seismic processes, might have occurred.

\section{Introduction}

For many years, research into the interaction between seismic activity and disturbances in radio broadcasts has been carried out. At first, results were obtained using $18 \mathrm{MHz}$ radio waves (Warwick et al., 1982) and later on, using the Omega and Loran radio waves that lie in the VLF (3$30 \mathrm{kHz}$ ) frequency band (Gokhberg et al., 1989; Hayakawa and Sato, 1994; Morgounov et al., 1994; Hayakawa et al., 1996 a, b; Molchanov and Hayakawa, 1998). In this same frequency band, seismogenic emission were observed (Nomikos and Vallianatos, 1996; Vallianatos and Nomikos, 1998). Among radio signals, low frequency (LF) radio signals lie in the band between $30-300 \mathrm{kHz}$. LF radio wave reception is characterised by ground-wave and sky-wave propagation modes. For distances greater than $500 \mathrm{~km}$, the contribution of the ground wave to the electric field strength is about ten times below that of the sky-wave contribution. In this case, the ground wave provides a weak but fairly stable signal, whereas the sky-wave signal is larger but greatly variable between day and night, and between winter and summer. In 1991, we designed and built a receiver that was able to measure the electric field strength of one LF frequency at field sites where the noise is very low. We selected the LF broadcasting station MCO located in France. The monitoring equipment is detailed in Bella et al. (1989). At the beginning of 1993, we put into operation receivers at two sites in central Italy, named AS and PE, located at a distance of 
Table 1. Parameters of the broadcasting stations

\begin{tabular}{cccc}
\hline Label & $\begin{array}{c}\text { Radiated Power } \\
(\mathrm{kW})\end{array}$ & $\begin{array}{c}\text { Frequency } \\
(\mathrm{kHz})\end{array}$ & $\begin{array}{c}\text { Mean distance } \\
\text { from receivers } \\
(\mathrm{km})\end{array}$ \\
\hline $\mathrm{CLT}$ & 60 & 189 & 540 \\
$\mathrm{MCO}$ & 1400 & 216 & 520 \\
$\mathrm{CZE}$ & 1500 & 270 & 820 \\
\hline
\end{tabular}

$60 \mathrm{~km}$ from each other. During 1996, we planned to record, in addition to the MCO signal, the signals of two other broadcasting stations: CZE (Czech Republic) and CLT (Sicily, in southern Italy). We built monitoring equipment similar to the previous system, but had the ability to measure the electric field strength of the three transmitters at the same time. At the end of 1996, we put into operation the new receivers at the original sites. The radiated power and frequency of the broadcasting stations, together with the mean distance from the receivers, are indicated in Table 1. In Fig. 1, the location of the transmitters and the receivers is shown. Some possible seismo-disturbances in these radio signals have been previously presented (Bella et al, 1998; Biagi, 1999; Biagi et al., in press). In this paper, we present the anomalous case records revealed so far, and the possibility that they represent precursors of earthquakes.

\section{Results}

Figure 2a shows the two anomalous decreases in the electric field strength of the MCO signal detected at the AS site using the original (MCO-only) system. The duration of the attenuation is 11 days (upper plot) and 5 days (lower plot), and the extent is up to $15 \mathrm{~dB}$. No anomaly at the PE site was detected at that time. About 15 days after the end of the 1993 radioanomaly, an earthquake with $\mathrm{M}=3.5$ occurred at $25 \mathrm{~km}$ from the receiver, along the MCO-AS path; furthermore, 4 days after the end of the 1994 radio-anomaly, a seismic sequence happened along the MCO-AS path at about $40 \mathrm{~km}$ from AS, with a magnitude of 3.0-3.5. The location of these seismic events is shown in Fig. $2 b$ and their time of occurrence is indicated in Fig. 2a.

With the new equipment for receiving the MCO, CLT and CZE transmissions, we again observed attenuation with an extent of $10-15 \mathrm{~dB}$ in the radio signals lasting several days, as shown in Figs. $3 \mathrm{a}$ and $4 \mathrm{a}$. In both cases, the attenuation appeared on just the CLT signal; the first one (Fig. 3a) was detected only at the PE site and the second one (Fig. 4a) at the AS site. Some days after the end of these radio-anomalies, earthquakes with magnitudes of 3.5 and 3.3 occurred at distances less than $50 \mathrm{~km}$ from the receivers, just along the transmitter-receiver path. The location of these earthquakes is shown in Figs. $3 b$ and $4 b$ and their time of occurrence is indicated in Figs. 3a and 4a. Many well-known effects con-

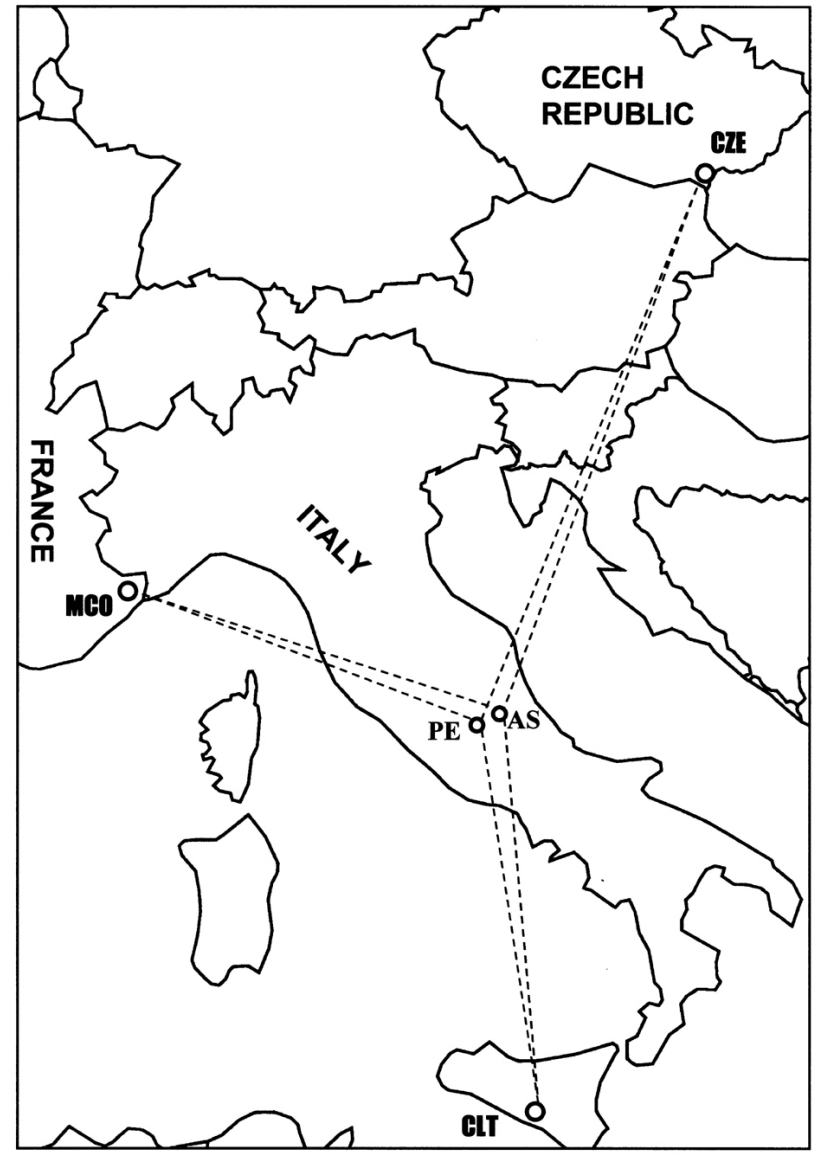

Fig. 1. Map showing the radio broadcasting stations (CLT, MCO, $\mathrm{CZE}$ ) and the measurement sites (AS, PE).

nected with a pre-seismic phase (electromagnetic emissions, gas emanation and ionisation) could create ducts, reflecting layers or scattering zones in trophosphere. These cause irregularities in the radio refractivity $\mathrm{N}$ that may be capable of producing a defocusing of the radio signals (CCIR, 1990a), as we have observed. The next step was to examine all of the data recorded since January 1997, i.e. from the beginning of the measurements with the new equipment, up to March 2000. First, a 7-day high pass filter was applied to the raw data to remove the high frequency components and then the long-term smoothed trend of the filtered data was determined by fitting a 9th order polynomial. Next, we created a working file as the difference between each filtered set and its polynomial fit. Finally we calculated the standard deviation $\sigma$ over each entire work data set. Plots of the CLT, MCO and CZE radio signals obtained at the AS site are shown in Fig. 5a. The horizontal dashed lines in each series represent the $\pm 3 \sigma$ level. If an anomaly is considered to be a value occurring over $3 \sigma$, then from Fig. $5 \mathrm{a}$ it is possible to note that: (1) no anomaly appears on the MCO data file; (2) three anomalies appear on the CLT plot during summertime as a quasiperiodic effect; (3) an anomaly appears on the CZE trend during February - March 1998. Turning first to the CLT anoma- 

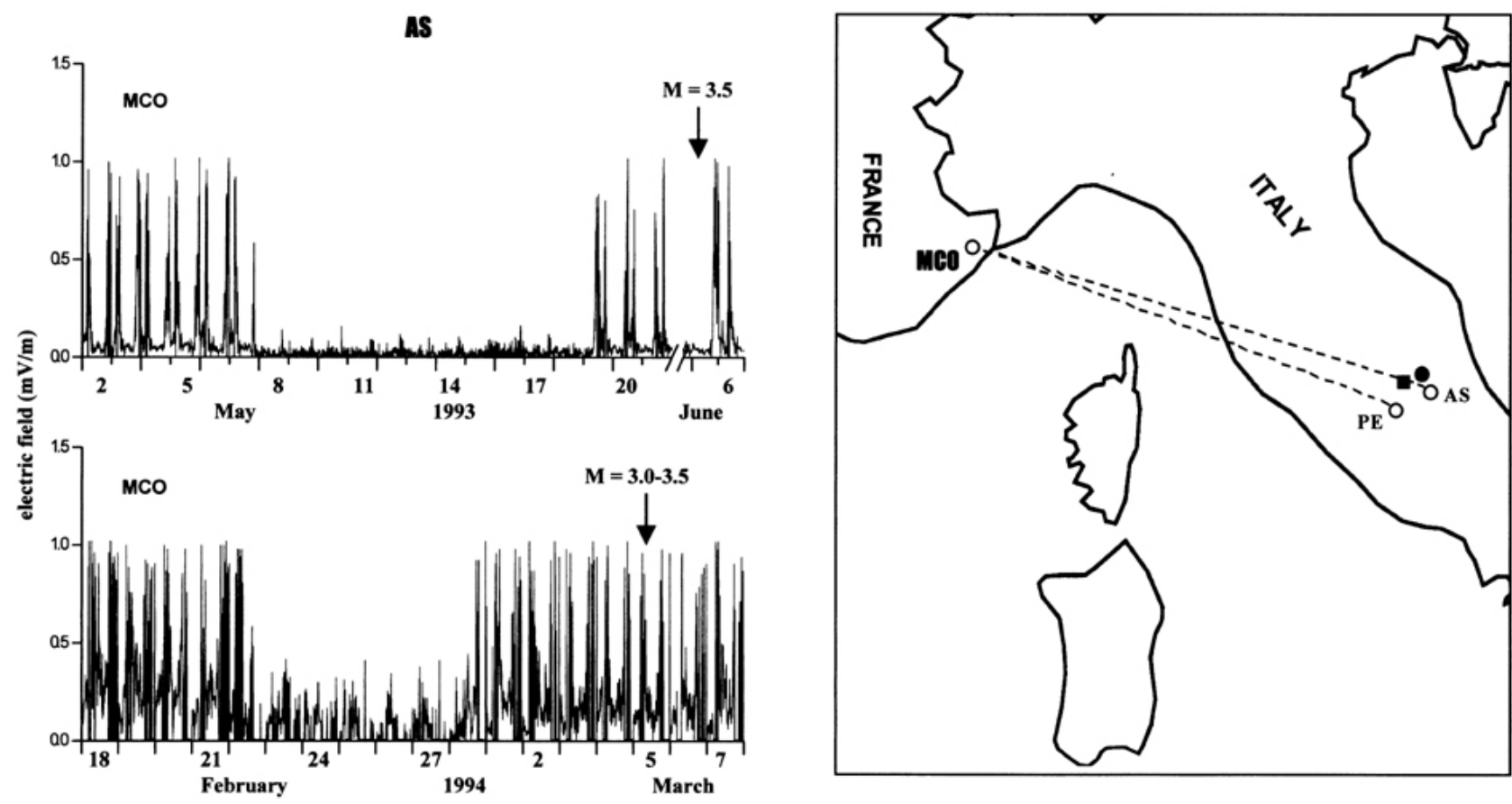

Fig. 2. (a) Anomalous decreases in the MCO electric field strength at the AS measurement site occurring in May 1993 and February 1994. The arrows indicate the earthquake $(M=3.5)$ that occurred on 5 June 1993 and the seismic sequence $(M=3.0-3.5)$ that occurred on 4 and 5 March 1994. (b) Map showing the location of the 1993 earthquake (black circle) and of the 1994 seismic sequence (black square).

lies, it was found that the broadcasting station increased the radiated power during the anomalous periods. In contrast, however, the CZE anomaly is not thought to be related to the broadcasting station. This anomaly is an increase of the order of $6-8 \mathrm{~dB}$ and in the middle of March 1998, i.e. practically at its end, a strong seismic crisis started in Slovenia $(\mathrm{M}=5.2$ on 13 March; $M=6.0$ on 12 April; $M=5.1$ on 6 May), in a zone lying in the middle of the transmitter-receiver path. It must be noted that according to the wave hop propagation theory (Knight, 1973), the sky-wave signal received by an antenna can be considered as a ray starting from the transmitter and reflected one or more times (hops) by the lower ionosphere and by the ground. The propagation of the skywave is thus controlled (CCIR, 1990b) by several parameters including the elevation angle $\psi$ (relative to the horizontal) of departure and the arrival of the ray, the ionospheric reflection coefficient $R$, the height $H$ of the ionosphere, the transmitting and receiving antenna factors $F$ and the ionospheric focusing factor $D$. Of these, $R$ is the main parameter of interest and the large daytime attenuation of the sky-wave with respect to the nighttime propagation conditions is primary caused by the different $R$ values. For example, $R$ takes typical values of around 0.01 during the day and 0.1 during the night for one hope wave. Therefore, it seems reasonable that the pre-seismic phase of the previous seismic crisis could have produced a variation of $R$ related to one hope mode of CZE radio signal and this variation could justify the anomaly we have observed. The location of the seismic crisis is shown roughly in Fig. 5b and the time of occurrence is indicated in
Fig. 5a. Unfortunately, the data recorded by the other receiver (PE) were affected by problems with both the power supply and the saturation of the recorded signals, so we could not use the data in this study of the whole data set.

\section{Discussion}

Our analysis is concerned with the observations of the variations in the radio signal strengths, and so we can focus on just the sky-wave signal component. First, consider the observed anomalous decreases in the radio signals. Initially, the meteorological conditions in central Italy were investigated and it was noted that during the anomalous time intervals, some thermal inversions in the low troposphere appeared. However, these meteorological conditions are fairly frequent in central and southern Italy and occurred several times during the experimental period with a duration of several days, without producing any decrease in the radio signals. As a result, a direct relationship between the observed radio-anomalies and meteorological phenomena does not seem satisfactory. The next step was to check for a possible correlation to the seismic activity at that time. Other seismic events were identified, having the same energy release that occurred along the transmitter-receiver paths at distances less than $50 \mathrm{~km}$ from the receivers, but in these cases, no field strength anomalies of the radio signals were found. Thus, a simple relationship between radio-anomalies and seismicity raises some doubtful points. However, an important negative result was noted; when the seismic events occurred with no radio-anomalies, 

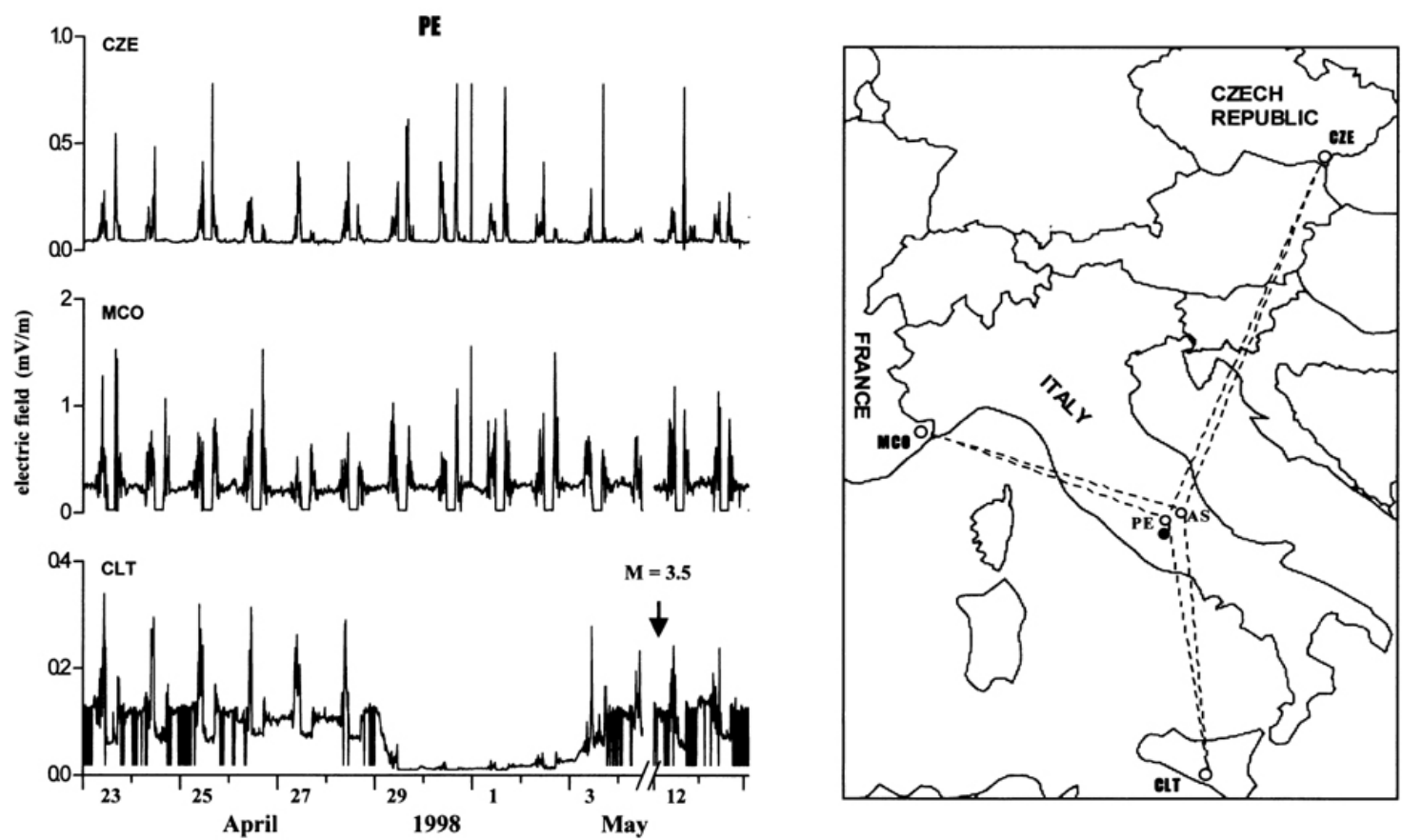

a)

b)

Fig. 3. (a) Anomalous decreases in the CLT electric field strength at the PE measurement site occurring in April - May 1998. The arrow indicates the earthquake $(M=3.5)$ that occurred on 12 May 1998 along the CLT-PE path. (b) Map showing the location of the earthquake (black circle).
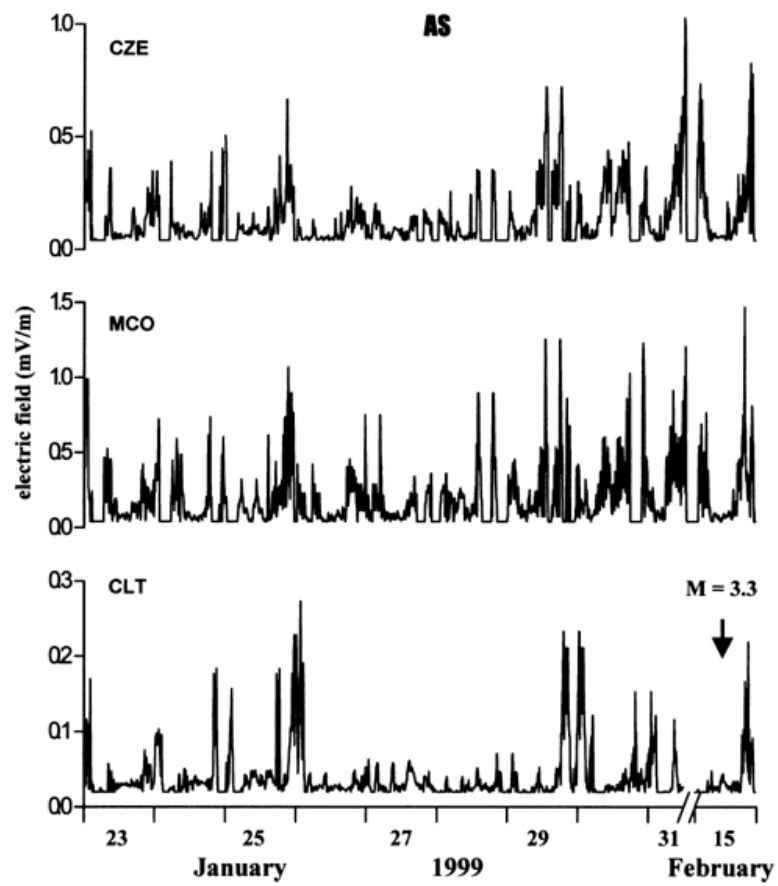

a)

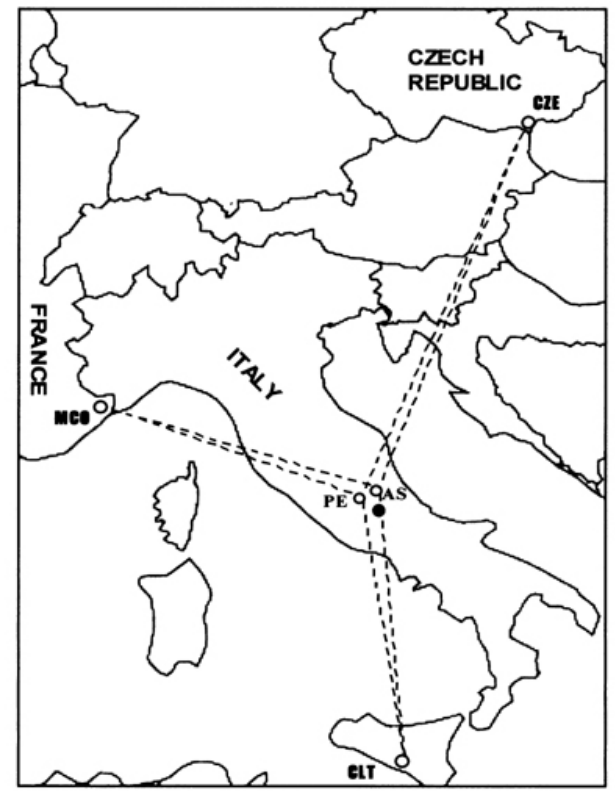

b)

Fig. 4. (a) Anomalous decreases in the CLT electric field strength at the AS measurement site occurring in January 1999. The arrow indicates the earthquake $(M=3.3)$ that occurred on 15 February 1999 along the CLT-AS path. (b) Map showing the location of the earthquake (black circle). 


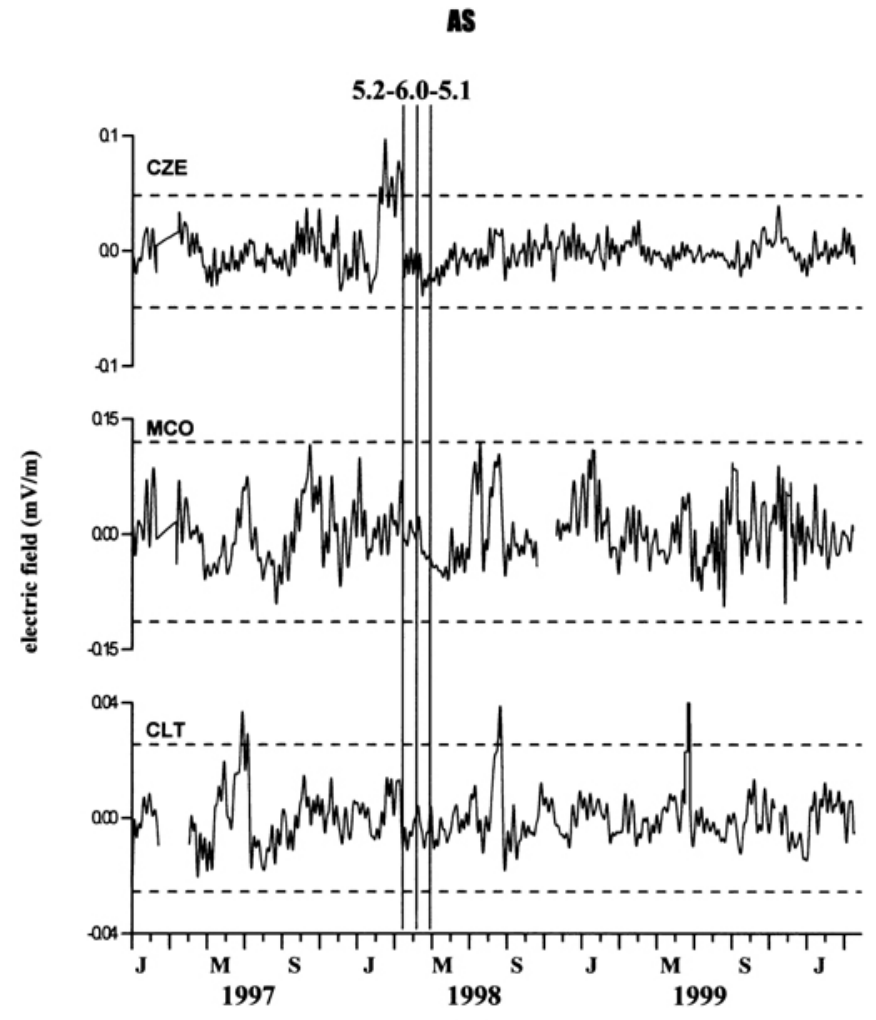

a)

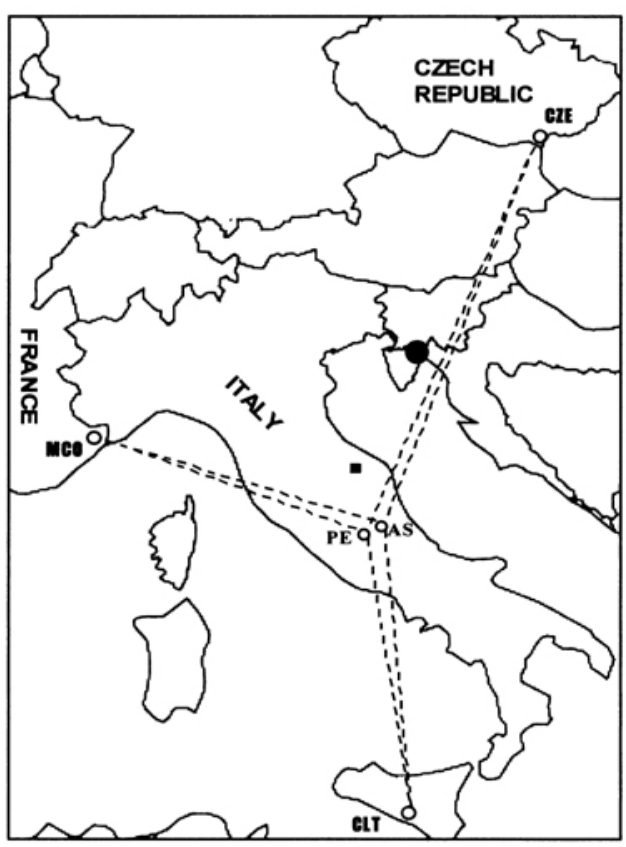

b)

Fig. 5. (a) Difference between the filtered data and smoothed trends of the CLT, MCO and CZE radio signals at the AS site from 1 January 1997 to 31 March 2000. The horizontal dashed lines represent the $\pm 3 \sigma$ level (where $\sigma$ is the standard deviation). The vertical lines represent the occurrence of the main earthquakes $(M=5.2,13$ March 1998; $M=6.0,12$ April 1998; $M=5.1,6$ May 1998) in the Slovenia region. (b) Map showing roughly the location of the Slovenia earthquakes (black circle). The location of the Umbria-Marche earthquake $(\mathrm{M}=5.3$ ) occurred on 26 March 1998 is also indicated (black square) on the map.

the meteorological analysis revealed no thermal inversion connected to the advection of warm air. Therefore, this result seems to indicate that the anomalous decreases we revealed are connected to concurrent or simultaneous phenomena: the preparatory phase of earthquakes and the advection of warm air in the troposphere.

The anomalies presented seem to fit with some defocusing process of radio signals in the troposphere since the epicentres are located where the sky-wave is already in the troposphere. Within a normal meteorological framework, the irregularities produced by the seismic processes as described in Sect. 2, do not normally act on LF radio signals but suitable conditions, such as warm air advection, might permit the diffusion of the irregularities over a sufficiently largescale to influence the LF radio wave propagation. In general, the warm air advection acts as a catalyst for the influence of the irregular variations of the radio refractivity produced by pre-seismic effects on the LF radio signals. Our observations were related only to moderate earthquakes and in these cases, the suitable meteorological conditions were probably needed as a catalyst to observe the effect. For larger earthquakes, it seems unnecessary for such coincident phenomena to be needed.
Consider now the anomalous increases in the CZE radio signal observed during February - March 1998. The first observation is that since it was only observed on the CZE signal, it means that the effect occurred only in the northeastern part of the map shown in Fig. 1. This increase of around 6-8 dB in the recorded radio signal might be explained by a $2-3$ times increase in the value of $R$ in the reflection point zone of the one hop wave, which is the principle sky-wave propagation mode. The pre-seismic phase of the Slovenian seismic crisis, the epicentres of which are 100-200 km away from the previous reflection point, might have produced such an increase in the $R$ value. It must be noted that distances of $100-200 \mathrm{~km}$, related to earthquakes of $\mathrm{M}=5.0-6.0$, are in agreement with the existence of the pre-seismic perturbed ionospheric region as suggested by Ruzhin and Depueva (1996) on the basis of different experimental results.

At the XXV EGS Assembly (Biagi et al., in press), some of the authors of this paper proposed that the anomaly under investigation was a precursor of the earthquake $(M=5.3)$ that occurred in the Umbria-Marche region on 26 March 1998, the location of which is indicated in Fig. 5b. This possibility cannot be excluded, but some doubts exist. First, the Umbria-Marche seismic sequence started on 26 September 
1997 with two strong earthquakes $(\mathrm{M}=5.6, \mathrm{M}=5.9)$ and lasted for several months. We must question why a premonitory anomaly appeared before the March 1998 final strong shock but not before the September 1997 main shocks. Second, the March 1998 earthquake was characterised by a large focal depth $(\approx 50 \mathrm{~km})$, but seismo-ionospheric perturbations might be expected only for shallow earthquakes (Molchanov and Hayakawa, 1998) and so the proposed precursor is very unusual. Finally, the March 1998 earthquake is located over $300 \mathrm{~km}$ from the reflection point of the one hope wave and so the perturbed ionospheric zone seems to be too large. Given these doubts about associating the anomaly with the UmbriaMarche earthquakes, a more convincing case is made in this paper in relating the anomaly to the Slovenia seismic crisis.

\section{Conclusions}

During eight years of measurements, we observed four anomalous decreases and one anomalous increase in the electric field strength of the LF radio signals. After the decreases, moderate $(3.0 \leq \mathrm{M} \leq 3.5)$ earthquakes occurred near the receivers (within $50 \mathrm{~km}$ ) along the transmitter-receiver path and, as a concomitant phenomenon, the advection of warm air occurred during the anomalous periods. After the increase, a strong ( $M=5.0-6.0$ ) seismic crisis occurred in Slovenia at more than $400 \mathrm{~km}$ from the receivers, but in the middle of the transmitter-receiver path. We advance the hypothesis that anomalies involving decreases are due to modifications of the troposphere and anomalies involving increases are caused by modifications of ionosphere, both related to the preparatory phase of earthquakes. Of course more observations are needed since on the basis of a few observations, one can only tentatively suggest that these are premonitory anomalies. But if this pre-seismic behaviour of the LF signals could be confirmed, then this type of precursor would seem capable of providing information on the direction, and perhaps even the rough location of a forthcoming earthquake.

\section{References}

Bella, F., Bella, R., Biagi, P. F., Della Monica, G., Ermini, A., Manjgaladze, P., Sgrigna, V., and Zilpimiani, D.: A digital recording system of electromagnetic emissions, Nuovo Cimento, 12C, 251-259, 1989.

Bella, F., Biagi, P. F., Caputo, M., Cozzi, E., Della Monica, G., Ermini, A., Plastino, W., and Sgrigna, V.: Field strength variations of LF radio waves prior to earthquakes in Central Italy, Phys. Earth Planet. Int., 105, 279-286, 1998.

Biagi, P. F.: Seismic Effects on LF Radiowaves, in: Atmospheric and Ionospheric Electromagnetic Phenomena Associated with Earthquakes, (Ed) Hayakawa, M., TERRAPUB, Tokyo, 535542, 1999.

Biagi, P. F., Ermini, A., and Kingsley, S. P.: Disturbances in LF radiosignals and the Umbria-Marche (Italy) seismic sequence in 1997-1998, Physics and Chemistry of the Earth, in press, 2001.

CCIR,: Effects of tropospheric refraction on radio-wave propagation, Rep., 718-3, 149-188, 1990a.

CCIR,: Sky-wave propagation and circuit performance at frequencies between about $30 \mathrm{kHz}$ and $500 \mathrm{kHz}$, Rep., 265-7, 212-229, $1990 b$.

Gokhberg, M. B., Gufeld, I. L., and Liperovsky, V. L.: Modern investigations of the electromagnetic prediction of the earthquakes, in: Discrete Properties of Geophysical Medium, Nauka Moscow, (in Russian), p. 92, 1989.

Hayakawa, M. and Sato, H.: Ionospheric perturbations associated with earthquakes, as detected by subionospheric VLF propagation, in: Electromagnetic Phenomena Related to Earthquake Prediction, (Eds) Hayakawa, M. and Fujinawa, Y., TERRAPUB, Tokyo, 391-397, 1994.

Hayakawa, M., Molchanov, O. A., Ondoh, T., and Kawai, E.: The precursory signature effect of the Kobe earthquake on VLF subionospheric signals, J. Comm. Res. Lab., 169-180, 1996a.

Hayakawa, M., Molchanov, O. A., Ondoh, T., and Kawai, E.: Anomalies in the subionospheric VLF signals for the 1995 Hyogo-ken earthquake, J. Phys. Earth, 44, 413-418, 1996 b.

Knight, P.: MF propagation: a wave-hop method for ionospheric field-strength prediction. BBC Eng., 10, 22-34, 1973.

Molchanov, O. A. and Hayakawa, M.: Subionospheric VLF signal perturbations possibly related to earthquakes, J. Geophys. Res., 103, 17 489-17 504, 1998.

Morgounov, V. A., Ondoh, T., and Nagai, S.: Anomalous variation of VLF signals associated with strong earthquakes (M geq 7.0), in: Electromagnetic Phenomena Related to Earthquake Prediction, (Eds) Hayakawa, M. and Fujinawa, Y., TERRAPUB, Tokyo, 409-428, 1994.

Nomikos, K. and Vallianatos, F.: Electromagnetic variations associated with the seismicity of the frontal Hellenic Arc, Geologica Carpathica, 49/1, 57-60, 1996.

Ruzhin, Yu. Y. and Depueva, A. Kh.: Seismoprecursors in space as plasma and wave anomalies, J. Atmos. Electr., 16, 271-288, 1996.

Vallianatos, F. and Nomikos, K.: Seismogenic radioemissions as precursors to earthquakes in Greece, Phys. Chem. Earth, 23, 938-944, 1998.

Warwick, J. W., Stoker, C., and Meyer, T. R.: Radio emission associated with rock fracture: possible application for the Great Chilean Earthquake of 22 May 1960, J. Geophys. Res, 87, 28512859, 1982. 